\title{
Prevalencia de sobrepeso, obesidad e hipertensión arterial en adolescentes de una escuela de arte
}

\author{
Prevalence of overweight, obesity and hypertension \\ in adolescents attending an art school
}

\author{
Patricia Torres \\ Jorgelina Linares ${ }^{1}$ \\ Natalia Bonzi 2 \\ Maria Elena Giuliano ${ }^{3}$ \\ Laura Sanziani ${ }^{3}$ \\ Stella M. Pezzotto 2,4 \\ Marta Bravo Luna ${ }^{4}$
}

1 Escuela Secundaria y de Arte $\mathrm{N}^{\circ} 4$ Nigelia Soria. Rosario. Argentina.

2 Universidad Nacional de Rosario. Facultad de Ciencias Médicas. Rosario. Argentina.

\section{Instituto de Cardio- logía-Hospital Italiano Garibaldi de Rosario. Argentina.}

4 Universidad Nacional de Rosario. Consejo de Investigaciones. Rosario, Argentina.

Recibido en 21-11-2010 Aceptado en 31-03-2011
Resumen - Se realizó un estudio analítico de corte transversal en la población de alumnos que concurren a una Escuela Pública Secundaria y de Arte ( $n=213$, varones 24\%, mujeres $76 \%$ ) en sus dos trayectos vocacionales (No-físico: Artes Visuales/Música y Físico: Danzas). Se determinaron variables antropométricas, presión arterial y frecuencia cardíaca. Se aplicó una encuesta sobre antecedentes personales de enfermedades crónicas no-transmisibles; traumatismos; ciclo menstrual; actividad física extraescolar; sedentarismo y horas de sue-

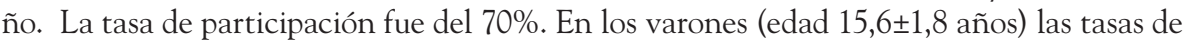
prevalencia de bajo peso, eutrofismo, sobrepeso y obesidad fueron de $0 \%, 87,5 \%, 12,5 \%$ y $0 \%$, respectivamente. En las mujeres (edad 15,5 $\pm 1,7$ ) estas tasas fueron $1,1 \%, 86 \%, 8,6 \%$ y $4,3 \%$. El índice de masa corporal se encontró significativamente asociado con los perímetros de cintura y braquial tanto en varones como en mujeres $(p<0,001)$. En el grupo de alumnos con Sobrepeso/Obesidad se detectaron 2 adolescentes con PAS aislada, centilo 90. Un joven eutrófico con PAS/PAD centilo 90 fue confirmado como "borderline" por presurometría de 24h. En las mujeres con Sp/Ob se detectaron 2 alumnas con PAS aislada centilo 90, una con PAD aislada centilo 90 y dos con PAS/PAD centilo 90. El estado nutricional de los alumnos es satisfactorio, con alta proporción de jóvenes eutróficos en ambos sexos. Sin embargo, la aplicación de este protocolo permitió detectar jóvenes con valores elevados de PA así como jóvenes con sobrepeso/obesidad. Dada la actividad escolar realizada por estos alumnos, estos factores podrían constituir un riesgo para su salud.

Palabras clave: Actividad motora; Antropometría; Estado nutricional; Obesidad Sobrepeso.

Abstract - A cross-sectional analytical study involving the population of adolescent students attending the Nigelia Soria Public School and Art Institute ( $n=213,24 \%$ boys, 76\% girls) in the two career paths (non-physical: visual arts and music, and physical artistic activities: dance) was conducted. Anthropometric variables, blood pressure (systolic, SBP, and diastolic, DBP), and heart rate were measured. A semi-structured questionnaire collecting personal data regarding non-communicable chronic diseases, trauma, menstrual cycle, non-school physical activity, inactivity, and sleep duration was administered. The participation rate was 70\%. In boys (age $15.6 \pm 1.8$ years), the prevalence rates of low weight, eutrophy, overweight, and obesity were $0 \%, 87.5 \%, 12.5 \%$ and $0 \%$, respectively. In girls (age $15.5 \pm 1.7$ years), these rates were $1.1 \%$, $86 \%, 8.6 \%$, and $4.3 \%$. Body mass index was significantly associated with waist circumference and brachial circumference in both genders $(p<0.001)$. In the overweight/obesity group, two students were diagnosed with isolated systolic hypertension (SBP 90th percentile). A eutrophic male student with SBP/DBP $90^{\text {th }}$ percentile was confirmed as borderline by 24 -h blood pressure measurement. In the group of overweight/obese girls, two students were identified with isolated $\mathrm{SBP} 90^{\text {th }}$ percentile, one with isolated DBP $90^{\text {th }}$ percentile, and two with SBP/DBP $90^{\text {th }}$ percentile. The nutritional status of students is satisfactory, with a high proportion of young healthy adolescents of both genders. However, the implementation of this protocol permitted to identify adolescents with high blood pressure, overweight, and obesity. These factors may pose a health risk considering the school activity of these students.

Key words: Anthropometry; Nutritional status; Obesity; Overweight; Physical activity. 


\section{INTRODUCCIÓN}

Rosario es uno de los principales centros urbanos de Argentina. La Escuela Pública Secundaria Provincial de Arte Nigelia Soria (ENS), es única en su tipo tanto en Rosario como en el resto del País, reuniendo en una sola institución la escolaridad obligatoria y la carrera artística.

Para la OMS, la salud no es solamente la ausencia de enfermedad, sino un estado de completo bienestar físico, mental y social. La nutrición es una de las necesidades básicas de salud para que se desarrolle al máximo el potencial biológico.

Se consideran adolescentes a los jóvenes entre 10-19 años. El rápido crecimiento de estatura, masa muscular y masa grasa en la adolescencia eleva los requerimientos dietarios, originándose diferencias en los puntos de corte que definen su estado nutricional ${ }^{1-11}$. La creciente independencia, la mayor participación en la vida social y las múltiples ocupaciones de los adolescentes influyen negativamente sobre sus hábitos alimentarios, constituyéndolos en un grupo de riesgo para desarrollar problemas de malnutrición. A esto se suman en esta escuela, los entrenamientos físicamente exigentes de los trayectos de danza, con necesidades energéticas aumentadas.

La malnutrición es carencia, exceso o desequilibrio en la ingesta de macro y micronutrientes, con dos polos: desnutrición y sobrealimentación. La sobrealimentación es un estado crónico con ingesta alimentaria superior a las necesidades energéticas, generando Sobrepeso (Sp) u Obesidad (Ob). Esta última es una condición compleja multifactorial, con componente tanto genético como ambiental, caracterizada por porcentaje anormalmente elevado de grasa corporal, generalizado o localizado ${ }^{1,2,7,8,10}$. Los desórdenes nutricionales son frecuentes en jóvenes sometidos a actividades físicas de alto rendimiento ${ }^{12-15}$, pudiendo desencadenar patologías en el aparato locomotor ${ }^{16-18}$, trastornos del ciclo menstrual por el deficiente o desbalanceado aporte de macro y micronutrientes que producen alteraciones en la producción de hormonas sexuales o la ocurrencia de anemia ferropénica. La malnutrición predispone también a graves alteraciones cardíacas en corazón estructuralmente sano ${ }^{19,20}$, desconocidas por el adolescente o sus familiares. En este último caso, si bien existe la presencia de genes específicos admitidos en la aparición de alteraciones electro fisiológicas cardíacas, se admite que las alteraciones nutricionales, especialmente deficitarias llevan a una labilidad del sistema nervioso autónomo que favorece su desencadenamiento, con la ocurrencia de hasta muerte súbita.

Si bien en la provincia de Santa Fe, en el año 2006 fue promulgada la ley No.12226 de obligatoriedad de examen integral y auditivo-visual de los escolares de Nivel Inicial y Educación General Básica, sólo se exige la presentación de certificado de profesionales médicos haciendo constar que el alumno es apto para la realización de actividades de educación física curricular no competitiva. En establecimientos escolares con exigencias mayores, como la ENS, los adolescentes quedarían sin las correspondientes medidas de prevención para ciertas patologías cuya morbi-mortalidad se ha incrementado.

En el presente trabajo se estudia a los alumnos de esta escuela con características especiales en cuanto a las exigencias físicas, no comprendidas en la ley anteriormente citada, con el fin de conocer su capital de salud, promoviendo la misma y previniendo la aparición de hábitos nocivos y de factores de riesgo.

\section{PROCEDIMIENTOS METODOLÓGICOS}

Diseño - Estudio analítico de corte transversal Población - Alumnos matriculados (año 2008) de ambos sexos que concurren a la ENS, quienes fueron invitados a participar por medio de notas dirigidas a padres y/o tutores, adjuntándose formulario de consentimiento informado y de aceptación del alumno, según normas de la Declaración de Helsinki ${ }^{21}$.

Se estudiaron los alumnos de las dos ofertas educacionales: obligatoria y vocacional con sus trayectos de artes visuales y música (sin exigencias físicas especiales), denominado Trayecto no-físico $(\mathrm{TnF})$ y de danzas (con alto rendimiento), denominado Trayecto físico (TF).

\section{Técnica e instrumentos de recolección de datos Antropometría}

Peso y Talla: con balanza de báscula, provista de tallímetro. Se calculó el índice de masa corporal (IMC) mediante el programa EpiInfo 2003, que calcula el valor de IMC e IMC centilado (IMCc) utilizando como referencia las curvas para edad, sexo, altura y peso de CDC/OMS. Para el IMCc, se considera bajo peso (Bp) si es menor a 5, eutrofismo (E) entre 5 y 85, sobrepeso (Sp) por encima del percentil 85 y obesidad $(\mathrm{Ob})$ por encima del percentil $95^{2,3}$. Según los valores de IMC se considera Bp si es menor a 18 , E entre 18 y 24,9; Sp entre 25 y 29,9 
y $\mathrm{Ob}$ si $\mathrm{IMC} \geq 30^{2}$. En este trabajo los estudiantes fueron clasificados según sus valores de IMC.

Perímetro braquial $(\mathrm{Pb})$ : Determinado en el punto medio de la longitud del brazo medida desde el acromion a la parte saliente del olécranon.

Perímetro de cintura $(\mathrm{Pc})$ : Determinado en la parte media tomando como punto de referencia la décima costilla y la porción más saliente de la cresta ilíaca, para determinar adiposidad central.

\section{Medición de presión arterial (PA) y frecuencia cardíaca (FC)}

Presión arterial Sistólica (PAS) y diastólica (PAD) medidas con esfigmomanómetro de mercurio en ambiente tranquilo, temperatura agradable y con reposo previo de 5 minutos, expresados en $\mathrm{mmHg}$ y con transformación a centilos. Categoría 1: normales (centilo 50) y Categoría 2: pre-hipertensión (centilo $\geq 90$ ). Según las tablas de PA en centilos ${ }^{22}$, para un joven que está en el centilo 95 de estatura el valor correspondiente al centilo 90 es de $136 \mathrm{mmHg}$ para PAS y $84 \mathrm{mmHg}$ para PAD. Para las mujeres los valores son $128 \mathrm{mmHg}$ y $81 \mathrm{mmHg}$, respectivamente. Se determinó FC por auscultación, durante 1 minuto en los intervalos de las lecturas de PA. Se realizaron 4 lecturas para PA y FC, utilizándose su promedio. A los alumnos con valores de PAS/PAD en el centilo 90, se los citó en dos oportunidades más, separadas por no menos de una semana ni más de dos, para confirmar el diagnóstico y derivarlo al cardiólogo del equipo. Se eligió como punto de corte el centilo 90 para realizar este algoritmo asimilando a la conducta preventiva que se realiza en adultos en el deseo de confirmar el diagnóstico de PA alta, sea PAS y/o PAD.

\section{Encuesta semi-estructurada}

Antecedentes personales - Ocurrencia de traumatismos, presencia de enfermedades crónicas no transmisibles (ECNT). En las jóvenes: edad de la menarca, características del ciclo menstrual, tratamiento hormonal y su causa.

Actividad física - Se evaluó la actividad física extraescolar (AFE), es decir aquella realizada fuera de la escuela y del horario escolar. Se utilizó el instrumento IPAQ, traducido y homologado para Argentina ${ }^{23}$ que considera intensidad (tipo de actividad), tiempo (minutos/día) y frecuencia (días/ semana). Se definieron las siguientes categorías: $0=$ no actividad; $1=$ baja actividad (menos de $30 \mathrm{~min}$ en menos de 3 días por semana; $2=$ moderada ( 30 min de 3 a 5 días por semana) y $3=a$ alta (más de 30 min en 5 o más días). Horas de sueño. Se tomó en cuenta sólo de lunes a jueves, se dividió en 2 categorías: $1 .<7$ h/día; $2 . \geq 7$ h/día.

Análisis Estadístico - Las variables cuantitativas se presentaron como Media \pm Desvío Estándar y se compararon mediante pruebas $t$ de Student o U de Mann-Whitney, según correspondiera. Se calcularon coeficientes de correlación (r) para evaluar la asociación entre variables antropométricas. Las variables cualitativas se presentan como frecuencias absolutas y porcentajes. Para la comparación de proporciones se utilizó la prueba Chi-cuadrado o la Prueba de Fisher, según correspondiera. Se consideraron significativas las pruebas con $\mathrm{p}<0,05$. Se utilizó el Software SPSS 11.0.

El proyecto fue aprobado por la Comisión de Bioética de la Facultad de Ciencias Médicas, Universidad Nacional de Rosario.

\section{RESULTADOS}

La matrícula 2008 fue de 213 alumnos. Aceptaron participar el $70 \%(\mathrm{n}=149), 36$ varones $(24 \%)$ y 113 niñas (76\%). No todas las mediciones antropométricas y/o encuestas previstas pudieron realizarse, debido a causas impredecibles durante el cursado.

Tabla 1. Variables antropométricas del total de varones y mujeres relevados

\begin{tabular}{lcccc}
\hline & \multicolumn{3}{c}{ Varones } & \multicolumn{2}{c}{ Mujeres } \\
\hline Variables & Media & Desvío estándar & Media & Desvío estándar \\
Edad (años) & 15,55 & 1,80 & 15,50 & 1,58 \\
Peso (kg) & 61,42 & 12,12 & 54,29 & 10,30 \\
Talla (cm) & 168,70 & 7,36 & 156,10 & 21,77 \\
IMC & 21,43 & 2,98 & 59,79 & 3,38 \\
IMCc & 58,81 & 23,90 & 70,05 & 23,24 \\
PC (cm) & 75,16 & 7,74 & 25,37 & 3,71 \\
Pb (cm) & 26,75 & 3,47 & & \\
\hline
\end{tabular}

IMC: índice de masa corporal, IMCc: índice de masa corporal centilado, Pc: perímetro de cintura,

Pb: perímetro braquial. 


\section{Antropometría}

Las variables antropométricas estratificadas por sexo se presentan en la tabla 1.

En los varones $(\mathrm{n}=24)$, se encontraron las siguientes tasas de prevalencia: Bp 0\%; E 87,5\%; Sp $12,5 \% ; \mathrm{Ob} 0 \%$. En varones eutróficos ( $\mathrm{n}=21$, edad $15,5 \pm 1,9$ años): $\mathrm{IMC}=20,6 \pm 2,2 ; \mathrm{IMCc}=54,0 \pm 21,4$. $P_{c}=73,5 \pm 6,0 \mathrm{~cm}$ y $\mathrm{Pb}=26,0 \pm 3,0 \mathrm{~cm}$.

En las mujeres $(\mathrm{n}=93)$, las tasas de prevalencia fueron: Bp 1,1\%; E 86,0\%; Sp 8,6\%; Ob 4,3\%. En mujeres eutróficas $(n=80$, edad $15,7 \pm 1,4)$ : $\mathrm{IMC}=20,6 \pm 1,8 ; \mathrm{IMCc}=53,5 \pm 28,6 ; \mathrm{P}_{\mathrm{c}}=68,1 \pm 4,9 \mathrm{~cm}$ y $\mathrm{Pb}=24,4 \pm 2,5 \mathrm{~cm}$.

No se encontraron diferencias entre sexos en las tasas de prevalencia de sobrepeso $(\mathrm{p}=0,400) \mathrm{ni}$ obesidad $(\mathrm{p}=0,394)$.

Las variables antropométricas estratificadas por trayecto y sexo se presentan en la tabla 2. No se encontraron diferencias estadísticamente significativas entre los valores medios de estas variables para cada trayecto, tanto en el sexo masculino como en el femenino.

En el sexo masculino el IMC se encontró significativamente correlacionado con $\mathrm{Pc}(\mathrm{r}=0,95$; $\mathrm{p}<0,001)$ y con $\mathrm{Pb}(\mathrm{r}=0,96 ; \mathrm{p}<0,001)$. En el sexo femenino, las correlaciones también resultaron significativas con $\mathrm{Pc}(\mathrm{r}=0,84 ; \mathrm{p}<0,001)$ y con $\mathrm{Pb}$ $(\mathrm{r}=0,73 ; \mathrm{p}<0,001)$. Se realizó el mismo análisis estratificando por trayecto, obteniendo los siguientes valores: Varones TnF: IMC-Pc $(r=0,93 ; p=0,001)$, IMC-Pb $(r=0,94 ; p<0,001)$. Varones TF: IMC-Pc $(\mathrm{r}=0,93 ; \mathrm{p}<0,001)$, IMC-Pb $(\mathrm{r}=0,97 ; \mathrm{p}<0,001)$. Mujeres TnF: IMC-Pc $(r=0,79 ; p<0,001)$, IMC-Pb $(\mathrm{r}=0,90 ; \mathrm{p}<0,001)$. Mujeres TF: IMC-Pc $(r=0,87$; $\mathrm{p}<0,001)$, IMC-Pb $(r=0,68 ; \mathrm{p}<0,001)$.

\section{Presión Arterial}

Se compararon los valores promedio de PAS y PAD (mmHg) y FC (latidos/min.) por sexo, y dentro de sexo según trayecto, no encontrándose diferencias significativas tanto en varones como en mujeres. Sólo la FC en mujeres resultó significativamente diferente entre las correspondientes a los distintos trayectos $(p=0,017)$. Estas diferencias se condicen con las explicaciones fisiológicas correspondientes al grupo etáreo en estudio.

Para PAS centilada se obtuvieron las siguientes proporciones: Categoría 1: varones $87 \%(\mathrm{n}=20)$ y mujeres $88 \%(\mathrm{n}=79)$; Categoría 2: varones $13 \%$ $(n=3)$; mujeres total $12 \%(n=11)$, sin diferencias significativas.

Para PAD centilada se obtuvieron las siguientes proporciones: Categoría 1: varones $70 \%(\mathrm{n}=16)$ y mujeres 79\% (n=71); Categoría 2: varones 30\% $(n=7)$ y mujeres $21 \%(n=19)$, sin diferencias significativas.

La estratificación por trayecto para PAS y PAD en varones no mostró diferencias significativas. En mujeres, la proporción de alumnas con PAS normal fue de $63,0 \%$ en las correspondientes al TF y $25,9 \%$ en las alumnas de $\operatorname{TnF}(\mathrm{p}=0,06)$.

En el grupo TF se detectó un joven eutrófico de 16 años calificado en centilo 90, con PAS y

Tabla 2. Variables antropométricas comparando Trayectos No-Físico (TnF) y Trayecto Físico (TF), según sexo.

\begin{tabular}{|c|c|c|c|c|c|c|c|}
\hline & \multicolumn{4}{|c|}{ Varones } & \multicolumn{3}{|c|}{ Mujeres } \\
\hline & Trayecto & $\mathrm{N}$ & Media & $\begin{array}{l}\text { Desvío } \\
\text { estándar }\end{array}$ & $\mathrm{N}$ & Media & $\begin{array}{l}\text { Desvío } \\
\text { Estándar }\end{array}$ \\
\hline \multirow[t]{2}{*}{ Edad (años) } & $\mathrm{TnF}$ & 11 & 15,12 & 1,93 & 24 & 16,06 & 1,46 \\
\hline & TF & 14 & 15,85 & 1,87 & 66 & 15,34 & 1,48 \\
\hline \multirow[t]{2}{*}{ Peso (kg) } & $\mathrm{TnF}$ & 8 & 60,36 & 12,50 & 21 & 53,41 & 8,48 \\
\hline & TF & 13 & 63,16 & 13,25 & 62 & 54,82 & 11,05 \\
\hline \multirow[t]{2}{*}{ Talla (cm) } & $\mathrm{TnF}$ & 8 & 168,04 & 7,79 & 21 & 157,66 & 8,20 \\
\hline & TF & 13 & 170,06 & 7,52 & 62 & 155,92 & 19,12 \\
\hline \multirow[t]{2}{*}{$\mathrm{IMC}\left(\mathrm{kg} / \mathrm{m}^{2}\right)$} & $\mathrm{TnF}$ & 8 & 21,18 & 2,72 & 21 & 21,46 & 2,61 \\
\hline & TF & 13 & 21,69 & 3,47 & 61 & 21,80 & 3,67 \\
\hline \multirow[t]{2}{*}{ IMCc } & $\mathrm{TnF}$ & 8 & 60,38 & 20,92 & 21 & 58,00 & 23,88 \\
\hline & TF & 13 & 59,29 & 28,56 & 61 & 59,84 & 23,20 \\
\hline \multirow[t]{2}{*}{$\mathrm{PC}(\mathrm{cm})$} & $\mathrm{TnF}$ & 8 & 76,33 & 8,25 & 21 & 70,70 & 5,12 \\
\hline & TF & 13 & 75,08 & 8,02 & 62 & 69,97 & 7,36 \\
\hline \multirow[t]{2}{*}{$\mathrm{Pb}(\mathrm{cm})$} & $\mathrm{TnF}$ & 8 & 26,83 & 3,00 & 21 & 25,19 & 2,77 \\
\hline & TF & 13 & 27,03 & 4,10 & 62 & 25,27 & 4,03 \\
\hline
\end{tabular}

IMC: índice de masa corporal, IMCc: índice de masa corporal centilado, Pc: perímetro de cintura, $\mathrm{Pb}$ : perímetro braquial.

Comparaciones entre grupos mediante pruebas $U$ de Mann-Whitney (diferencias no significativas). 
PAD 128/78 mmHg, respectivamente, que fue derivado al cardiólogo del equipo diagnosticándosele Presión Normal Alta mediante presurometría. Los 3 varones clasificados como Sp/Ob, 2 en TF y uno en $\mathrm{TnF}$, fueron derivados al nutricionista del equipo, uno de ellos (14 años) tenía PAS=128 $\mathrm{mmHg}, \mathrm{PAD}$ normal y $\mathrm{FC}=74$. El análisis de las jóvenes con $\mathrm{Sp} / \mathrm{Ob}$, derivadas al nutricionista, permitió detectar participantes que estaban en el centilo 90 de PA. Un estudio cardiológico más profundo no confirmó patología cardiovascular en estas alumnas.

\section{Antecedente Personales}

\section{- ECNT}

En los varones no se detectaron patologías en glándula tiroides, diabetes ni dislipidemias. El $7 \%$ de los varones presentó enfermedad cardiovascular. En el 6\% de las mujeres se detectó enfermedad cardiovascular, en el 5\% patología de glándula tiroides, en el $1 \%$ diabetes y en el $1 \%$ dislipidemias.

\section{- Antecedentes de traumatismos}

Habían sufrido traumatismos el 63\% de los varones y $49 \%$ de las mujeres. El $76 \%$ de los varones y $72 \%$ de las mujeres respondió que asistieron al médico o asistirían en caso de lesionarse.

- Ciclo menstrual.

La edad media a la menarca fue $12,1 \pm 1,2$ años; la duración del período fue $28,6 \pm 4,7$ días; la duración de la menstruación de 5,0 $\pm 4,7$ días. Se produjo interrupción en el 27\%. Administración hormonal (anticonceptivos orales) en el 16\%. Concurren al ginecólogo el 40\%.

- Horas de sueño promedio de lunes a jueves. El $37 \%$ de los varones y $51 \%$ de las mujeres acostumbraba dormir menos de $7 \mathrm{~h} / \mathrm{d}$.

- Actividad Física Extraescolar (AFE).

Practican AFE el 78\% de los varones y el 51\% de mujeres. El análisis de AFE según sexo y trayecto se muestra en la tabla 3. La proporción de alumnos con actividad baja $(\mathrm{IPAQ}=1)$ fue significativamente mayor $(p=0,014)$ en los varones $(71 \%)$ que en las mujeres (35\%). Tanto en los varones como en las mujeres la comparación de las proporciones entre trayectos (uniendo categorías 2 y 3 ) mostró fuerte significado estadístico ( $\mathrm{p}=0,002$ y 0,008 , respectivamente).
Tabla 3. Características de los alumnos según Actividad Física Extraescolar (AFE) comparando Trayectos No-Físico (TnF) y Trayecto Físico (TF), según sexo.

\begin{tabular}{lcccc}
\hline & $\begin{array}{c}\text { IPAQ 1 } \\
\mathrm{n}(\%)\end{array}$ & $\begin{array}{c}\text { IPAQ 2 } \\
\mathrm{n}(\%)\end{array}$ & $\begin{array}{c}\text { IPAQ 3 } \\
\mathrm{n}(\%)\end{array}$ & $\mathrm{p}^{*}$ \\
\hline Varones TnF & $32(88)$ & $4(12)$ & $0(0)$ & 0,002 \\
Varones TF & $20(55)$ & $16(45)$ & $0(0)$ & \\
Mujeres TnF & $76(67)$ & $37(33)$ & $0(0)$ & 0,008 \\
Mujeres TF & $20(18)$ & $89(79)$ & $4(3)$ & \\
\hline
\end{tabular}

IPAQ 1=baja actividad (menos de 30 min en menos de 3 días por semana; IPAQ 2= moderada (30 min de 3 a 5 días por semana) y IPAQ $3=$ alta (más de 30 min en 5 o más días).

*Combinando categorías IPAQ1 e IPAQ2

\section{DISCUSIÓN}

La ENS es única en su género en la ciudad de Rosario y en todo el País, debido a que proporciona a los alumnos la educación secundaria obligatoria y al mismo tiempo ofrece la oportunidad de desarrollar su vocación en el arte. Según la legislación vigente en nuestra provincia, se exige a los alumnos entre 5 y 14 años la presentación de certificado de salud habilitando para realizar actividades de educación física curricular no competitiva. El grupo constituido por los adolescentes de 15 años hasta el egreso escolar se convierte en un "grupo desprotegido", en especial si asisten a establecimientos escolares con exigencias físicas mayores, como la ENS.

A través de este estudio queremos mostrar la importancia de la realización de un monitoreo riguroso. La implementación de nuestro protocolo no está enmarcada sólo en la recolección de datos sino en obtener un acercamiento con el joven y responder a sus dudas. Todos los jóvenes son informados de los valores obtenidos y en caso de detectar alguna alteración se cita a los padres/tutores para informarles y asesorarlos.

El estudio del estado nutricional a través de las variables antropométricas permitió conocer la escasa prevalencia de bajo peso en este grupo, así como la correspondiente a las categorías sobrepeso y obesidad, en comparación con otros estudios que muestran tasas superiores para ambas categorías ${ }^{2-6,9-11}$. Es de destacar el alto porcentaje de jóvenes eutróficos en el grupo estudiado, que permite inferir su buen estado nutricional.

En la actualidad no existen estándares aprobados internacionalmente para perímetro de cintura, sin embargo los resultados aquí presentados son coincidentes con los comunicados en la literatura ${ }^{24,25}$.

Teniendo en cuenta las exigencias relacionadas al trayecto físico se decidió estratificar a los jóvenes según el mismo, ya que los valores de las 
variables antropométricas en este grupo podrían ser distintos, debiendo sistematizarse a fin de detectar problemas nutricionales ${ }^{12-15}$. Es importante destacar además que a pesar de que los alumnos no son bailarines profesionales, su antropometría está muy cercana a la requerida en esos casos ${ }^{14,15}$.

La definición de sobrepeso/obesidad es un tema muy controvertido en cuanto a los puntos de corte que se deben tomar, así como los estándares que se utilizan. Ha surgido también la necesidad de que el diagnóstico de Sp/Ob sea simple y confiable, pues si bien se puede recurrir a pruebas más sofisticadas tales como medición de pliegues, impedancia bioeléctrica, absorción dual de rayos X o aplicación de ecuaciones para valorar la masa grasa ${ }^{7,26}$, éstas requieren la utilización de tecnología costosa y preparación técnica del operador, encareciendo el diagnóstico o llevándolo al terreno del especialista. Es por eso que en el Congreso de Obesidad 2007 en Hungría ${ }^{27}$ éste fue uno de los temas prioritarios. El profesional de atención primaria debe estar preparado para diagnosticar $\mathrm{Sp} / \mathrm{Ob}$ de una forma sencilla y eficiente. Atendiendo a este propósito se realizó análisis de correlación del IMC con las variables $\mathrm{Pc}$ y $\mathrm{Pb}$, obteniéndose un altísimo significado, confirmando la utilidad de estas sencillas medidas antropométricas para un diagnóstico de Sp/Ob.

Según aconseja la Fourth Task Force ${ }^{22}$ se convirtieron los valores de PAS y PAD a centilos, no encontrándose diferencias significativas entre sexos. Es de notar que tanto los valores de PAS como PAD están de acuerdo a los que fisiológicamente se admiten según sexo e intervalo etáreo. No obstante, la aplicación de este protocolo permitió detectar jóvenes con valores de PA que debieron ser derivados al cardiólogo para su control.

Los antecedentes personales de ECNT fueron escasos en ambos sexos. Con respecto a la conducta del adolescente frente a traumatismos, tanto en el caso de haberlos padecido o ante la posibilidad de sufrirlos, un alto porcentaje de alumnos respondió que asistieron al médico o asistirían en caso de lesionarse. Es decir que el cuidado corporal, relacionado estrechamente al estado nutriciona ${ }^{16-19}$, es importante para ellos.

El conocimiento de las características del ciclo menstrual es relevante, ya que está influenciado fuertemente por la nutrición. El 98\% de las niñas ha tenido la menarca, siendo el promedio de edad 12 años, levemente más bajo que el actualmente admitido fisiológicamente ${ }^{28-30}$. Las características del ciclo menstrual se encuentran dentro de los patrones normales en las adolescentes.
Las horas de sueño tienen gran importancia, pues significan ahorro de energía. Sin embargo sólo el 63\% de los varones y 49\% de las mujeres duermen 7 o más horas diarias.

Este efectivo protocolo puede ser desarrollado por personal de las áreas de educación física, capacitado para realizar ciertas prácticas diagnósticas no especializadas pero que permiten realizar un seguimiento de la salud de los alumnos y detectar la aparición de posibles factores de riesgo, para así sugerir una conducta apropiada o, en caso de ser necesario, solicitar la participación de especialistas, siempre con la anuencia de los padres o tutores.

Insistiendo en la aplicación de este minucioso protocolo no invasivo y de bajo costo, consideramos sería de interés aplicarlo en otras escuelas en las que existen prácticas deportivas competitivas, sometiendo a los alumnos a rigurosos entrenamientos sin un control como el aquí propuesto.

\section{CONCLUSIONES}

El estado nutricional de los alumnos es satisfactorio, con alta proporción de jóvenes eutróficos en ambos sexos. Sin embargo, la aplicación de este protocolo permitió detectar jóvenes con valores elevados de PA así como jóvenes con sobrepeso/ obesidad. Dada la actividad escolar realizada por estos alumnos, estos factores podrían constituir un riesgo para su salud.

\section{Agradecimientos}

Los autores agradecen a la Directora Sra. Beatriz Ferrer, a la Vicedirectora Sra. Silvia Aparicio, al cuerpo docente y al personal de la Escuela Secundaria No.4 e Instituto de Arte Nigelia Soria de Rosario.

Agradecimiento también a la Secretaría de Ciencia,Tecnología e Innovación, Facultad de Ciencias Médicas, UNR, por el subsidio recibido ( $\left.\mathrm{N}^{\circ} 869 / 2008\right)$.

\section{REFERENCIAS BIBLIOGRÁFICAS}

1. Daniels SR, Arnett DK, Eckel RH, Gidding SS, Hayman LL, Kumanyika S, et al. Overweight in Children and Adolescents: Pathophysiology, Consequences, Prevention, and Treatment. Circulation 2005;111:1999-2012.

2. Cole TJ, Bellizzi MC, Flegal KM, WH. Establishing a standard definition for child overweight and obesity worldwide: international survey. BMJ 2000;320:1-6.

3. Giugliano R, Melo ALP. Diagnosis of overweight and obesity in schoolchildren: utilization of the body mass index international standard. J Pediatr 2004;80(2):129-34. 
4. Kovalskys I, Bay L, Rausch HC, Berner E. Prevalencia de obesidad en una población de 10 a 19 años en la consulta pediátrica. Arch Pediatr Urug 2004;75(4):345-51.

5. Del Pino M, Bay L, Lejarraga H, Kovalskys I, Berner E, Rausch HC. Peso y estatura de una muestra nacional de 1971 adolescentes de 10 a 19 años: las referencias argentinas continúan vigentes. Arch Argent Pediatr 2005;103(4):323-31.

6. Azcona San Julián C, Romero Montero A, Bastero Miñón P, Santamaría Martínez E. Obesidad Infantil. Rev Esp Obes 2005;3(1):26-39.

7. Moreno LA, Blay MG, Rodríguez G, Blay VA, Mesana MI, Olivares JL, et al. Screening Performances of the International Obesity Task Force Body Mass Index Cut-Off Values in Adolescents. J Am Coll Nutrition 2006;25(5):403-8.

8. Hall DMB, Cole TJ. What use is the BMI? Arch Dis Child 2006;91:283-6.

9. Coelho R, Sous S, Laranjo MJ, Monteiro AC, Bracança G, Carreiro H. Excesso de Peso e Obesidade Prevenção na Escola. Acta Med Port 2008;21:341-4.

10. Wieting MJ. Cause and Effect in Childhood Obesity: Solutions for a National Epidemic. Med J Am Osteopath Assoc 2008;108:545-52.

11. Cole TJ, Flegal KM, Nicholls D, Jackson AA. Body mass index cut offs to define thinness in children and adolescents: international survey. Br Med J 2007;335:194-7.

12. Betancourt León H, Albizu Campos JC. Estimación Antropométrica de la Composición Corporal en Bailarines de la Escuela Nacional de Ballet. Rev Biol 2005;19:1-2

13. Cosette M, Rodríguez M. Estado nutricional y orientación nutricional en estudiantes de ballet de nivel elemental. (Parte III). Trastornos de la Conducta Alimentaria 2008;7:903-26.

14. Betancourt León H, Aréchiga Viramontes J, Díaz Sánchez ME, Ramírez García CM. Valoración Antropométrica de la Composición Corporal de Bailarines de Ballet. Un estudio longitudinal. Rev Bras Cineantropom Desempenho Hum 2008;10(2):115-22.

15. Vázquez Sánchez V, Martínez Fuentes AJ, Carrillo Estrada U, Santos Beneit MG, Mesa Santurino MS, Marrodán Serrano MD. Body composition and nutritional condition in cubans students of ballet. Nutr Clin Diet Hosp 2008;28(3):3-8.

16. Frusztajer NT, Dhuper S, Warren MP, Brooks-Gunn J, Fox RP.Nutrition and the incidence of stress fractures in ballet dancers. Am J Clin Nutr 1990;5 (1):779-83.

17. Bowling A. Injuries to dancers: prevalence, treatment, and perceptions of causes. Br Med J 1989;298:731-4.

18. Valentino R, Savastano S, Pasquale Tommaselli A, D'amore G, Dorato M, Lombardi G. The Influence of Intense Ballet Training on Trabecular Bone Mass, Hormone Status, and Gonadotropin Structure in Young Women. J Clin Endocrinol Metab 2001;86(10):4674-8.

19. Chillier JC, Peace K, Hulme A, Healy JC. MRI features of foot and ankle injuries in ballet dancers. Br J Radiol 2004;77:532-7.

20. Katzman DK. Medical Complications in Adolescents with Anorexia Nervosa: A Review of the Literature. Int J Eat Disord 2005;37(supl):52-9.
21. Declaración de Helsinki de la Asociación Médica Mundial. Principios éticos para las investigaciones médicas en seres humanos. Disponible en: http://www.wma.net/ es/30publications/10policies/b3/17c_es.pdf. [2010 jul 03].

22. The Fourth Report on the: Diagnosis, Evaluation, and Treatment of High Blood Pressure in Children and Adolescents. NIH Publication No. 05-5267. Originally printed September 1996 (96-3790) Revised May 2005.

23. Cuestionario Internacional De Actividad Física IPAQ: Formato Corto Autoadministrado De Los Últimos 7 Días Para Ser Utilizado Con Adultos Jóvenes Y De Mediana Edad (15- 69 años). Disponible en: http:// www.ipaq.ki.se. [2007 mar 23].

24. McCarthy HD, Ellis SM, Cole TJ. Central overweight and obesity in British youth aged 11-16 years: cross sectional surveys of waist circumference. Brit Med J 2003;326(7390):624-7.

25. Fernández JR, Redden DT, Pietrobelli A, Allison DB. Waist Circumference Percentiles in Nationally Representative Samples of African-American, EuropeanAmerican, and Mexican-American Children and Adolescents. J Pediatr 2004;45:439-44.

26. Moreno VM, Gómez Gandoy JB, Antoranz González MJ. Medición de la grasa corporal mediante Impedancia Bioeléctrica, pliegues cutáneos y ecuaciones a partir de medidas antropométricas. Análisis comparativo. Rev Esp Salud Pública 2001;75:221-36.

27. Bonzi N, Bravo Luna M. Comentarios Sobre el Congreso Europeo de Obesidad 2007. Rev Méd Rosario 2007;73:148-50.

28. Zurlo de Mirotti SL, Lesa AM, Barrón de Carbonetti M. Age of menarche. Secondary sex characteristics. Interrelation. Secular trend. Rev Fac Cienc Med Universidad Nacional Córdoba 1995;53(supl.): 7-15.

29. Lejarraga H, Sanchirico F, Cusminsky M. Age of menarche in urban Argentinian girls. Ann Hum Biol 1980; 7(6):579-81.

30. Lejarraga H, del Pino M, Fano V, Caino S, Cole TJ. Growth references for weight and height for Argentinian girls and boys from birth to maturity: incorporation of data from the World Health Organization from birth to 2 years and calculation of new percentiles and LMS values. Arch Argent Pediatr 2009;107(2):126-33.

\section{Dirección postal}

Marta Bravo Luna

Universidad Nacional de Rosario.

Consejo de Investigaciones

Santa Fe 3100. 2000 Rosario. Argentina

E-mail: mbravoluna@gmail.com 